\title{
Structural Analysis of the Stratum Corneum using EPR and EPR Imaging with Stable Spin Probes
}

\author{
Kouichi Nakagawa* \\ Division of Regional Innovation, Graduate School of Health Sciences, Hirosaki University, 66-1 Hon-Cho, Hirosaki 036-8564, JAPAN
}

\begin{abstract}
X-band (9.4 GHz) electron paramagnetic resonance (EPR) and electron paramagnetic resonance imaging (EPRI) were used for elucidating structural aspects of the stratum corneum (SC). We found that psoriasis vulgaris (PV)-SC has a less-ordered structure than that of the control SC, indicating the abnormal architecture of PV-SC. Different spectra were observed for the PV-SC. The three-line spectral pattern suggests that the 5-doxylstearic acid (5-DSA) is mobile or less rigid in the SC. The simulated order parameter $\left(\mathrm{S}_{0}\right)$ value obtained for 5-DSA in the SC was approximately 0.20 . The statistical analysis suggests that the value of the PV-SC is significantly smaller than that of the control $(p<0.01)$. Thus, we suggest that this EPR assay is of great use for evaluating SC function. In addition, the EPRI of various SC samples provides a useful image concerning SC status. A strong red image was observed for the PV skin. No red lesion region was observed in the control. The EPR images differentiated various sizes and number distribution concerning the disordered states in the SC.
\end{abstract}

Key words: EPR, skin, stratum corneum, EPR imaging, spin probe, psoriasis vulgaris

\section{Introduction}

Electron paramagnetic resonance $(\mathrm{EPR})$ spectroscopy is a particularly sensitive technique that can detect endogenous and exogenous radicals both in vitro ${ }^{1-3)}$ and noninvasively in vivo ${ }^{4)}$. EPR is useful for elucidating structural aspects of stratum corneum $(\mathrm{SC})^{1-3)}$. Non-invasive spectroscopic characterization of the outermost layer of the SC is an important subject in dermatology and cosmetology. The $\mathrm{SC}$, a heterogeneous structure comprising corneocytes embedded in the intercellular lamellar lipid bilayer, acts as the main epidermal barrier against chemicals, oxidative stress, and other invasive environmental factors. It also regulates transepidermal water loss (TEWL) to prevent dehydration of viable cells underneath the SC. Measurement of TEWL is the most common method to estimate the state of the skin surface. However, the TEWL does not provide morphological information regarding the lipid in the SC.

EPR measures an unpaired spin (free radical) in a sample at any temperature. The motion of the unpaired spin is affected by the radical moiety and appears in the EPR spectral pattern. Aliphatic spin probes (such as 5-doxylstearic acid) that contain the unpaired spin, permeate the intercellular lipid region of the $\mathrm{SC}^{11}$. The EPR spectral pattern is related to the aliphatic spin-probe moiety in the SC. The EPR-spin probe method is useful for elucidating structural aspects of the SC. The motion of spin probes in the SC will provide a useful index regarding its structure. The obtained structural value (ordering) will vary according to the probe moiety in the SC. The relatively high values are associated with restricted motions in the SC. EPR could provide a useful index regarding SC. Thus, we examined the morphological aspects of the SC in patients with psoriasis vulgaris $(\mathrm{PV})$.

$\mathrm{X}$-band (at $9.4 \mathrm{GHz}$ one of the microwave-frequency bands) electron paramagnetic resonance imaging (EPRI) can verify the location of the unpaired spin in a sample. Although X-band EPRI is the same as magnetic resonance imaging (MRI) in principle, the sample sizes and quantities are limited by the microwave frequency and cavity size ${ }^{4-6)}$. We performed $9.4 \mathrm{GHz}$ EPRI to identify the locations of structural abnormality of the $\mathrm{SC}$ in patients with $\mathrm{PV}$. PV is characterized by small, erythematous lesions, and red bumps that enlarge, inflame, and form scales. However, the available structural information regarding PV is limited. EPRI can potentially offer further quantitative insights into SC states.

\footnotetext{
*Correspondence to: Kouichi Nakagawa, Division of Regional Innovation, Graduate School of Health Sciences, Hirosaki University, 66-1 Hon-Cho, Hirosaki 036-8564, JAPAN

E-mail: nakagawa@ hirosaki-u.ac.jp

Accepted October 1, 2019 (received for review September 4, 2019)

Journal of Oleo Science ISSN 1345-8957 print / ISSN 1347-3352 online

http://www.jstage.jst.go.jp/browse/jos/ http://mc.manusriptcentral.com/jjocs

This is the review by the winner of 53rd Award for Prominent Studies, The Japan Oil Chemists' Society (JOCS).
} 


\section{K. Nakagawa}

The 9.4 GHz EPRI of PV-SC samples was performed to examine SC status. Spectral analyses using an EPR simulation differentiates the SC status. In addition, the EPR images of a particular SC sample with respect to SC diseases showed various magnitudes of disordered SC structures. The possible structural changes in the SC with respect to PV are also discussed.

\section{Experimental}

\subsection{Samples}

Five patients with typical PV $(n=5$ : 40 years in age, male; 74 years in age, male; 41 years in age, male; 47 years in age, female; 43 years in age, female) who had not received any treatment for 3 months prior to the study were examined. The control SC was taken from skin lesions on forearms and subjected to EPR assay. The samples were taken from normal persons $(n=5$ : 54 years in age, male; 55 years in age, male; 70 years in age, male; 34 years in age, female; 53 years in age, female) using a previously described method $^{3,7)}$. The first strip of the control SC was also examined. The Hirosaki University Internal Review Board approved all protocols used herein (2014-390).

The SC sample was pasted onto a glass plate $(\sim 7 \times 37$ $\mathrm{mm}^{2}$ ) using a drop of cyanoacrylate resin. Approximately 1 $\mathrm{mg}$ of the stripped SC sample is required for control measurements; however, in the case of PV-SC, the sample amount varies from patient to patient. The detailed sample incubation processes are described in previous papers ${ }^{3,7)}$. After rinsing with distilled water to remove excess spin probe, the SC sample was mounted on an EPR cell and measured using 9.4 GHz EPRI. The sample was pasted onto an EPR rod (with an outer diameter of $\sim 4.0 \mathrm{~mm}$ ) for imaging measurement or on a glass plate using a single drop of a cyanoacrylate resin. The sample portion of the rod was positioned in the center of the microwave cavity.

Spin probe reagents, 5-doxylstearic acid (5-DSA), 4-hydroxy-2,2,6,6-tetramethylpiperidin-1-oxyl (TEMPOL), and 1,1-diphenyl-2-picrylhydrazyl (DPPH) radicals were purchased from Sigma-Aldrich Japan K.K., and Nacalai Tesque, Inc. (Kyoto, Japan) and used as received.

A piece of the $\mathrm{SC}$ was incubated in an $\sim 50 \mu \mathrm{M}$ aqueous solution of the spin probe, 5-DSA, for approximately 60 $\min$ at $37^{\circ} \mathrm{C}$. After incubation, the SC samples were measured using a $9.4 \mathrm{GHz}$ EPR spectrometer. The spectra of the SC samples were analyzed using a simulated order parameter $\left(\mathrm{S}_{0}\right)^{8,9)}$. Sample preparation and EPR measurements are described in detail elsewhere ${ }^{3,7}$.

\subsection{Analyses using order parameter}

To quantitatively analyze the obtained EPR spectra, we performed EPR spectral simulation. The 5-DSA molecules in the SC lipid undergo ordering, which restrict the ampli- tude of the rotational motion. The overall orientation of the probe was evaluated using the nonlinear least-squares fitting program (NLLS) and can be expressed by the order parameter $\left(\mathrm{S}_{0}\right)$ as follows ${ }^{8)}$ :

$$
S_{0}=\left\langle\frac{1}{2}\left(3 \cos ^{2} \gamma-1\right)\right\rangle
$$

which measures the angular extent of the rotational diffusion of the nitroxide probe moiety. Gamma $(\gamma)$ is the angle between the rotational diffusion symmetry axis and the $z$-axis of the nitroxide axis system ${ }^{8,9)}$. The $z$ axis is that of the nitrogen $2 \mathrm{p}_{\mathrm{z}}$ atomic orbital and the $\mathrm{x}$ axis lies along the $\mathrm{N}-\mathrm{O}$ bond, as shown in Fig. 1. The microscopic ordering of the probe in the multilamellar lipid bilayer is characterized by the $\mathrm{S}_{0}$ value. In the simulation based on the experimental spectra, the following principal components, $A_{X X}, A_{Y Y}$, $\mathrm{A}_{\mathrm{ZZ}}=(0.66,0.55,3.45) \mathrm{mT}$ and $\mathrm{g}_{\mathrm{XX}}, \mathrm{g}_{\mathrm{YY}}, \mathrm{g}_{\mathrm{ZZ}}=(2.0086$, $2.0063,2.0025)$, were used for 5-DSA ${ }^{9)}$.

\subsection{EPR measurements}

A JEOL RE-3X 9.4 GHz EPR spectrometer (JEOL Ltd., Tokyo, Japan) was used for ordinary measurements. The system was operated at $9.4 \mathrm{GHz}$ using a $100-\mathrm{kHz}$ modulation frequency. All EPR spectra were obtained in a single scan. The typical EPR settings were as follows: microwave power, $5 \mathrm{~mW}$; time constant, $0.1 \mathrm{~s}$; sweep time, $4 \mathrm{~min}$; magnetic field modulation, $0.32 \mathrm{mT}$; and magnetic field sweep width, 5- $10 \mathrm{mT}$.

\subsection{EPRI measurements and data processing}

A commercially available JEOL RE-3X 9.4 GHz EPR spectrometer was modified for use as an EPR imager to include the attachment of magnetic-field gradient coils (Yonezawa Densen Ltd., Yonezawa, Japan) and their power supplies. For 9.4 GHz EPRI, x- and y-axes of gradient coils with anti-Helmholtz coil configurations were used. To avoid overheating, the gradient coils were cooled with water. Takasago BWS 60-5 bipolar power supplies (Takasago Ltd., Tokyo, Japan) were used.

We used 16 equal-angle-spaced projections obtained with a maximum gradient of $\sim 3.3 \mathrm{mT} / \mathrm{cm}$. The first-derivative EPR spectra were numerically integrated to obtain the corresponding absorption spectra. Before reconstruction, each projection was deconvolved using the measured zerogradient spectrum to improve the image resolution. The two-dimensional (2D) images were reconstructed using the back-projection algorithm from the EPR-IT software package from the Center for EPR Imaging In Vivo Physiology at the University of Chicago ${ }^{10)}$. Imaging data were processed in the MathWorks MATLAB ${ }^{\mathrm{TM}}$ environment.

Notably, the magnetic field of the EPR spectrometer was current controlled to perform EPRI. The field-based control is incompatible with the application of the magnetic field gradients. The instrument was controlled using SpecMan4EPR (FeMi Instruments LLC, USA) software. In addi- 


\section{Membrane Surface}

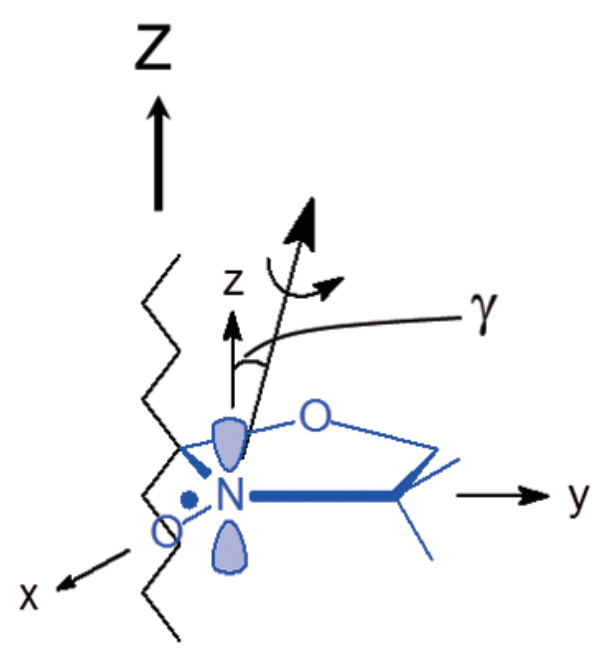

Fig. 1 A schematic representation of a conformation of DSA spin probe portion in the SC membrane, where Z-axis of the acyl chain is parallel to $\mathrm{Z}$-axis of the nitrogen $2 \mathrm{p}_{\mathrm{z}}$ orbital.

tion, images were acquired at room temperature using a Bruker E500 ELEXSYS system (Bruker BioSpin GmbH, Karlsruhe, Germany) equipped with a high-sensitivity TM resonator (10 $\mathrm{mm}$ in diameter, Bruker). The system was operated in X-band mode at $\sim 9.6 \mathrm{GHz}$ with a $100-\mathrm{kHz}$ modulation frequency. Detailed EPRI is described in previous papers ${ }^{5,6)}$. All measurements were performed at ambient temperature.

\section{Results and Discussion}

\subsection{X-band EPR of the stratum corneum}

Figure $2(\mathrm{~A})$ shows 5-DSA in aqueous solution. A sharp three-line signal of the 5-DSA aqueous solution was observed. The line shape suggests that molecular motion of 5 -DSA is rapid in the solution. Figure 2(B) shows the typical EPR spectrum of 5-DSA in PV-SC. A small, broad, three-line pattern was observed. This spectral pattern is quite different from those reported for other $\mathrm{SCs}^{1-3)}$. The spectral pattern suggests that 5-DSA is relatively mobile or less rigid in the SC. The red dashed-line is the simulated spectrum. Reasonable agreement between the experimental and simulated spectra was obtained as shown in Fig. 2 (B). The $\mathrm{S}_{0}$ value obtained for 5-DSA in the SC was approximately 0.20 . Notably, the lower value of $\mathrm{S}_{0}$ indicates the less rigid (abnormal) structure of the PV-SC. The dashed lines and the asterisks indicate rigid components of the spectra.

Figure 2(C) shows the EPR spectrum of the mid-volar forearm(control). The EPR pattern is very similar to that for the forearm SC previously reported ${ }^{1-3)}$. The red dashed-line is the simulated spectrum. Good agreement between the experimental and simulated spectra was also obtained. The simulated $\mathrm{S}_{0}$ value obtained was 0.40 . The quantitative structural ordering (0.40) of the SC lipids also implies that the probe moiety is relatively rigid. In addition, the signal intensity of the control is weaker than that of the PV-SC and does not show the strong three-line pattern. The weak signal demonstrates the low amount of 5-DSA permeation in the SC because of the rigid structure of the SC.

Figure 3 shows the bar chart of the $\mathrm{S}_{0}$ values corresponding to the control and the PV-SC. The low $\mathrm{S}_{0}$ value for the PV-SC is associated with the abnormal structure of the SC lipids. Student's t-test suggests that the value of the PV-SC is significantly smaller than that of the $\operatorname{control}(p<$ 0.01). Thus, the abnormal SC structure is implied for the PV-SC.

Next, we attempted to reproduce the PV-SC spectrum (Fig. 2(B)). Figure 4 shows extreme cases of the PV-SC and control SC. Panels (a) and (b) show the EPR spectrum of PV-SC-1 and control SC-1, respectively. In each case, the peak areas (double integrals) of each spectrum are normalized to 1 to perform a spectral addition. If the EPR spectrum of 5-DSA shows a three-line pattern, the 5-DSA probe is mobile. Contrarily, the EPR spectrum of 5-DSA is an immobile pattern. The spectrum in (c) is similar to that of Fig. 2 (B) except for the broad line width. The line-width shown in Fig. 4(A) is sharp because 5-DSA was originally mobile in the sample. Contrarily, the 5-DSA is immobilized and the EPR spectrum shows a broad asymmetric pattern. These spectral differences reflect the structural differences in the SC samples. Thus, the added spectrum in Fig. 4(C) suggests that approximately $30 \%$ of the 5 -DSA in the sample is in a rigid state.

PV is classified as a disorder of keratinization although its pathogenesis has not been fully elucidated. We typically recognize tick scale in psoriasis lesions and hyperkeratosis and parakeratosis are histologically found. The turnover time of psoriatic keratinocytes decreases approximately seven times and the increase in the proliferating cell components in the epidermis may cause differentiation abnormalities in keratinocytes. In fact, many results concerning differentiation abnormalities including an increase in the main keratin of epidermal cells and a decrease in profilaggrin expression have been found in psoriatic epidermis ${ }^{11-14)}$. In the EPR study, we found that the PV-SC has a less-ordered structure than that of the control SC, indicating the abnormal architecture of PV-SC. The EPR result is consistent with previous observations. Therefore, we suggest that the EPR assay is of great use for evaluating SC function. 


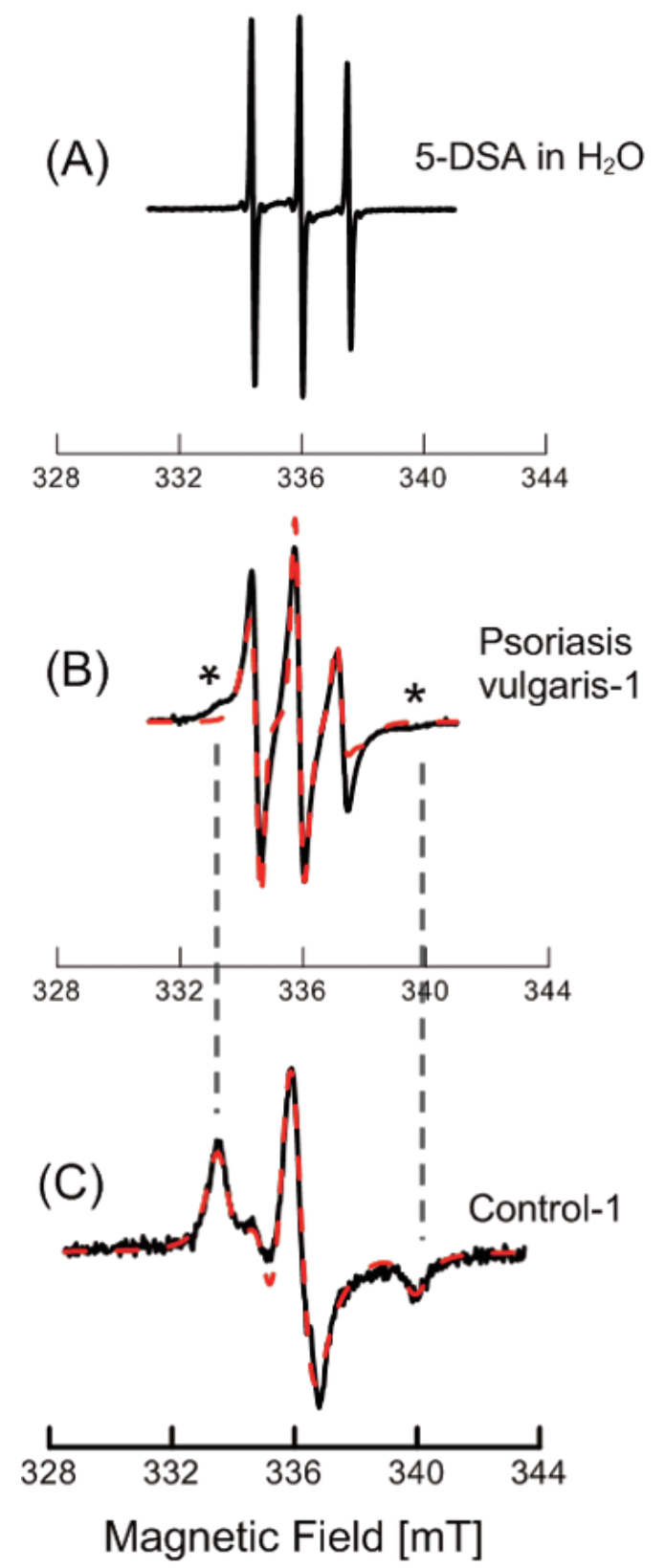

Fig. 2 (A) EPR spectrum of aqueous 5-DSA stock solution is presented. (B) EPR spectrum of (B) psoriasis vulgaris (PV) SC is presented. (C) EPR spectrum of 5 -DSA of the typical mid-volar forearm SC (control)is presented. Experimental (solid line) and simulated (dashed line) EPR spectra of 5-DSA probe are shown. All EPR spectra were obtained with the single scan. The dashed lines and the asterisks indicate rigid(immobilized) components of the spectra.

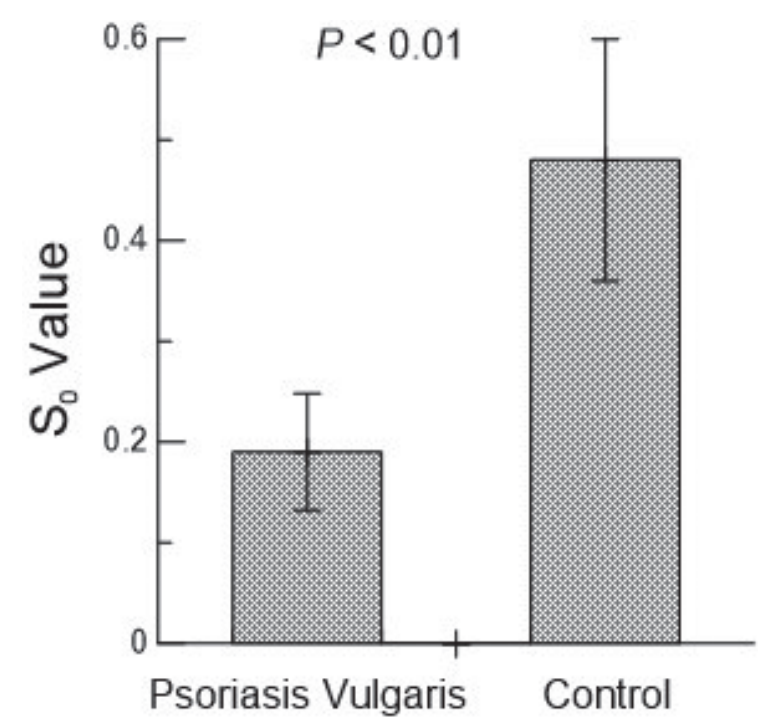

Fig. 3 Plot of simulated order parameter $\left(\mathrm{S}_{0}\right)$ of the control and the PV-SC. The statistical results obtained for the control SC and the PV-SC are 0.49 \pm 0.076 and $0.20 \pm 0.035$, respectively. Each value represents mean \pm SD of five measurements. The $\mathrm{S}_{0}$ values of the control SC show significantly higher values than that of the PV-SC $(p<0.01)$.

(a)
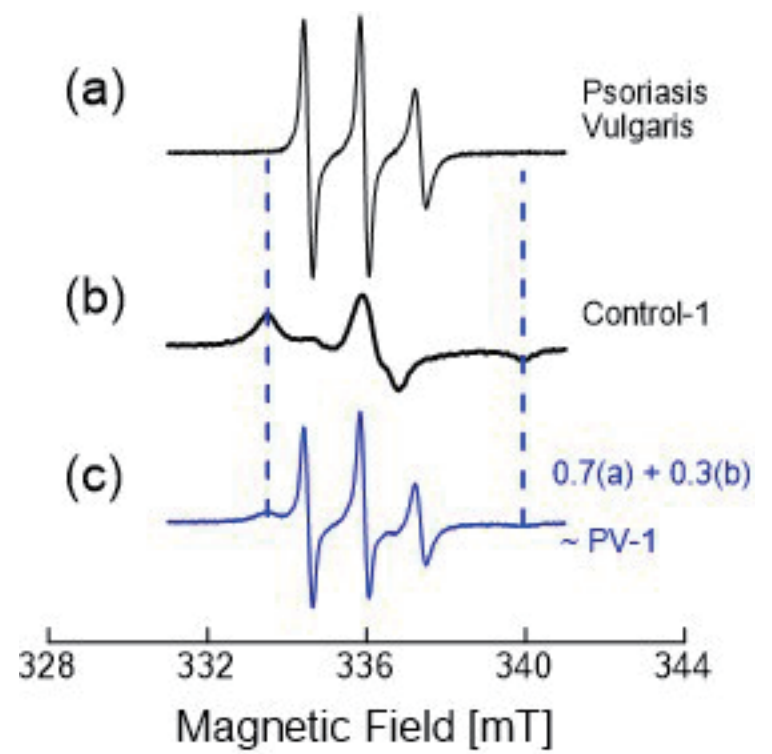

Fig. 4 (a) EPR spectrum of the PV-SC is presented. (b) EPR spectrum of the control SC is presented. The splitting $\left(\mathrm{A}_{\mathrm{zz}}\right)$ for the outer extremes is $6.49 \mathrm{mT}$. (c) Added EPR spectrum of $(0.7 \times(a)+0.3 \times(b))$ is presented. The dashed lines indicate immobilized components of the spectra. The distance of dashed lines is equal to $2 \mathrm{~A}_{\|}$(parallel). The spectrum is a reproduction of the spectrum of Fig. 2(B). 
(A)

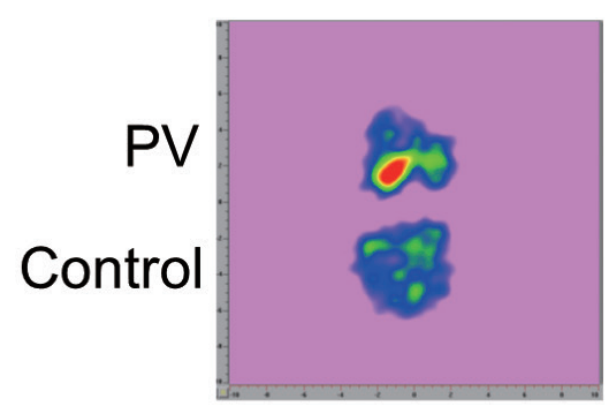

(B)

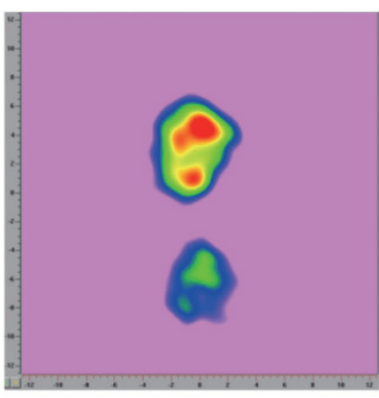

(C)

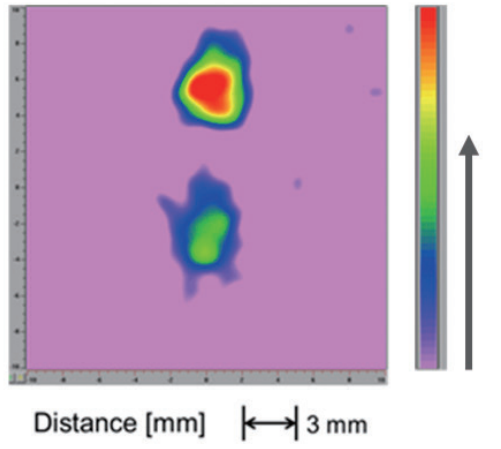

Fig. 5 Three imaging sets (A-C); each image shows the PV-SC (top) and control SC (bottom) samples. The axes units are also indicated.

\section{Normal}

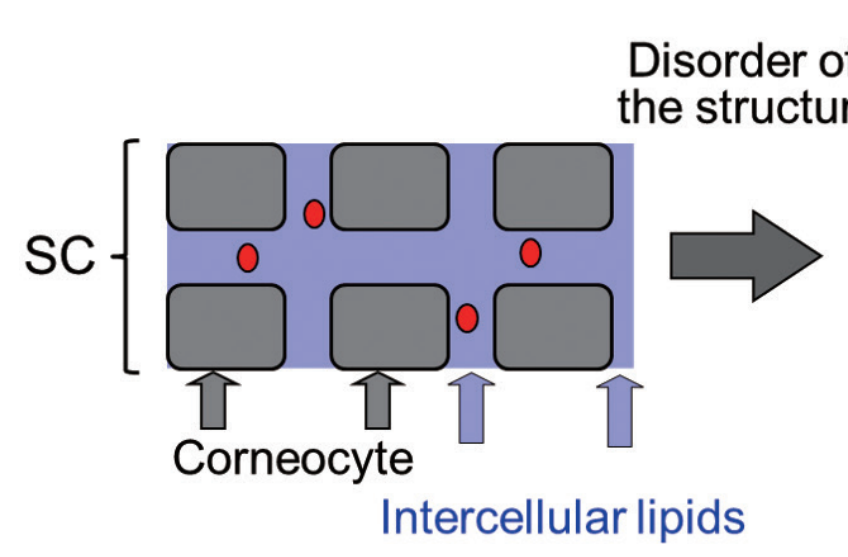

Abnormal

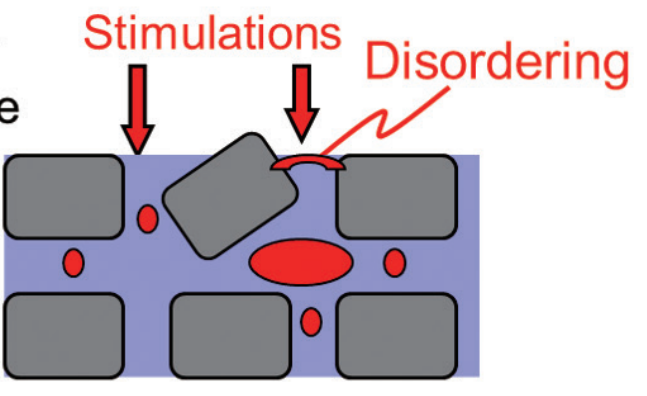

0 : Spin probe

Fig. 6 Schematic illustration of a possible structural disordering mechanism of stratum corneum (SC).

\subsection{EPRI of stratum corneum}

To further compare the structure of the SC to that of the PV-SC, we performed EPRI studies. Figure 5 shows three EPR images of the PV(top part) and control(bottom part) samples. Based on the linewidth of 5-DSA, the spatial resolution of the EPRI was approximately $0.11 \mathrm{~cm}$. The color and size of the skin image depend on the area of probe incubation because, typically, skin repels an aqueous solution. We simultaneously performed measurements on the $\mathrm{PV}$ and control samples to clearly compare the differences in the SC states. A red lesion region was observed in each PV sample. A relatively large red region was also observed in some cases.

A larger amount of probe per unit area of skin is distributed in the PV-SC sample. In addition, the narrowing of the EPR line width derived from the mobility increase in the probe molecule (5-DSA) should affect the EPR image intensity. Notably, two red regions were detected in the case of Fig. 5(B). The strong red signal is because of the probe penetration into the PV skin. Although the EPR spectra were similar to the control spectral pattern, the small lesion region of the PV skin may not be structurally uniform compared with that of the control.

No red lesion region was observed in the control; however, blue and partly green regions did occur. The results suggest that the probe molecule is uniformly distributed in the skin and that the probe moiety is rigid.

As we previously mentioned, we examined the ordinary EPR measurements. The results showed that the EPR spectra of the PV-SC were similar to those of the forearm SC previously reported ${ }^{1-3)}$. This spectral pattern suggests that the probe motion is somewhat rigid. Although the ordinary EPR spectra showed that the probe motion is restricted, the present EPRI showed that the probe distribution in the skin may be fairly uniform. While the EPR spectra of the PV patients are not similar to those of the controls, the limited state of the PV-SC becomes more comparable to that of the controls. However, the thicker 


\section{K. Nakagawa}

SC of the patients may have introduced probe penetration. Thus, the present EPR images demonstrate that the SC states of the control are more uniform than those of the PV-SC. The EPR images show various sizes and number distributions concerning the disordered states in the SC.

Figure 6 shows a schematic illustration of the PV-SC model based on the present results. The SC (left-hand side), a heterogeneous structure composed of corneocytes embedded in the intercellular lamellar lipid bilayer, acts as the main epidermal barrier to oxidative stress and other invasive environmental factors and regulates TEWL to prevent dehydration of viable cells underneath the SC.

When the rigidity of the structure decreased for various reasons, the structure of the SC becomes disordered (righthand side). The disordered structure of the SC can have areas that easily penetrate molecules, such as topically applied chemicals. The penetration of 5-DSA resulted in stronger EPR intensity. The relatively mobile 5-DSA provides EPR three lines. In addition, we obtained a red intense color for the PV-SC compared to that of the control. These observations are consistent with the schematic illustration of the SC structure. The disordering of the SC structure can directly lead to skin diseases.

The studied $9.4 \mathrm{GHz}$ EPRI can be useful for detecting and identifying the location of an abnormality of the SC state. In addition, the EPRI technique can be applied to other SC diseases. Therefore, X-band EPRI and EPR spectroscopy are very useful techniques that can be applied in evaluations.

\section{Acknowledgments}

The author thanks Prof. Daisuke Sawamura and Dr. Satoko Minakawa for the gifts of various samples and useful discussion about the EPR results. Part of this research was supported by KAKENHI Grant number 18K19890 from the Japan Society for the Promotion of Science(JSPS).

\section{References}

1) Nakagawa, K.; Mizushima, J.; Takino, Y.; Kawashima, T.; Maibach, H.I. Chain ordering of stratum corneum lipids investigated by EPR slow-tumbling simulation. Spectrochim. Acta Part A 63, 816-20 (2006).

2) Nakagawa, K. Electron paramagnetic resonance investigation of stratum corneum lipid structure. Lipid 45, 91-96 (2010).
3) Nakagawa, K.; Minakawa, S.; Sawamura, D. Spectroscopic evidence of abnormal structure of psoriasis vulgaris stratum corneum. J. Dermatol. Sci. 65, 222-224 (2012).

4) Epel, B.; Halpern, H. J. Electron paramagnetic resonance oxygen imaging in vivo. Electron Paramagnetic Resonance. R. Soc. Chem. 23, 180-208(2013).

5) Nakagawa, K; Ohba, Y; Epel, B; Hirata H. A 9 GHz EPR imager for thin materials: application to surface detection. J. Oleo Sci. 61, 451-456(2013).

6) Nakagawa, K.; Minakawa, S.; Sawamura, D.; Hara, H. Skin surface imaging of psoriasis vulgaris by using an electron paramagnetic resonance spin probe. J. Dermatol. Sci. 81, 71-73(2016).

7) Nakagawa, K.; Minakawa, S.; Sawamura, D. Stratum corneum structure of psoriasis vulgaris investigated by EPR spin-probe method. Appl. Magn. Reson. 44, 941948 (2013).

8) Budil, D.E.; Lee, S.; Saxena, S.; Freed, J.H. Nonlinearleast-squares analysis of slow-motion EPR spectra in one and two dimensions using a modified LevenbergMarquardt algorithm. J. Magn. Reson. Ser. A 120, 155-189 (1996).

9) Ge, M.; Rananavare, S.B.; Freed, J.H. ESR studies of stearic acid binding to bovine serum albumin. Biochim. Biophys. Acta 1036, 228-236(1990).

10) http://epr-it.specman4epr.com/ The web describes the EPR-IT software.

11) Iizuka, H.; Takahashi, H.; Ishida-Yamamoto, A. Psoriatic architecture constructed by epidermal remodeling. J. Dermatol. Sci. 35, 93-99 (2004).

12) Takemoto, H.; Tamai, K.; Akasaka, E.; Rokunohe, D.; Takiyoshi, N.; Umegaki, N.; Nakajima, K.; Aizu, T.; Kaneko, T.; Nakano, H.; Sawamura, D. Relation between the expression levels of the POU transcription factors Skn-1a and Skn-1n and keratinocyte differentiation. J. Dermatol. Sci. 60, 203-205(2010).

13) Kim, B.E.; Howell, M.D.; Guttman-Yassky, E.; Gilleaudeau, P.M.; Cardinale, I.R.; Boguniewicz, M.; Krueger, J.G.; Leung, D.Y. TNF- $\alpha$ downregulates filaggrin and loricrin through c-Jun N-terminal kinase: role for TNF- $\alpha$ antagonists to improve skin barrier. J. Invest. Dermatol. 131, 1272-1279(2011).

14) Oyama, R.; Jinnin, M.; Kakimoto, A.; Kanemaru, H.; Ichihara, A.; Fujisawa, A.; Honda, N.; Masuguchi, S.; Fukushima, S.; Maruo, K.; Ihn, H. Circulating microRNA associated with TNF- $\alpha$ signaling pathway in patients with plaque psoriasis. J. Dermatol. Sci. 61, 209211 (2011). 